\title{
Carathéodory, Helly and the Others in the Max-Plus World
}

\author{
Stéphane Gaubert • Frédéric Meunier
}

Received: 20 July 2008 / Revised: 27 April 2009 / Accepted: 10 June 2009 / Published online: 7 July 2009 (C) Springer Science+Business Media, LLC 2009

\begin{abstract}
Carathéodory's, Helly's and Radon's theorems are three basic results in discrete geometry. Their max-plus or tropical analogues have been proved by various authors. We show that more advanced results in discrete geometry also have max-plus analogues, namely, the colorful Carathéodory theorem and the Tverberg theorem. A conjecture connected to the Tverberg theorem-Sierksma's conjecture-although still open for the usual convexity, is shown to be true in the max-plus setting.
\end{abstract}

Keywords Colorful Carathéodory's theorem · Max-plus convexity · Sierksma's conjecture · Tropical geometry · Tverberg's theorem

\section{Introduction}

There are three basic theorems in discrete geometry that play a central role in convexity: Carathéodory's theorem, Helly's theorem and Radon's theorem. We state them here for the sake of completeness.

Theorem 1 (Carathéodory's theorem) Suppose given $n \geq d+1$ points $\boldsymbol{x}_{1}, \boldsymbol{x}_{2}, \ldots, \boldsymbol{x}_{n}$ in $\mathbb{R}^{d}$ and a point $\boldsymbol{p}$ in the convex hull of $\left\{\boldsymbol{x}_{1}, \boldsymbol{x}_{2}, \ldots, \boldsymbol{x}_{n}\right\}$. Then there is a subset $I \subseteq\{1, \ldots, n\}$ of cardinality $d+1$ such that $\boldsymbol{p}$ is in the convex hull of $\left\{\boldsymbol{x}_{i} \mid i \in I\right\}$.

The first author has been partially supported by the joint CNRS-RFBR grant number 05-01-02807.

S. Gaubert

INRIA and CMAP, École Polytechnique, 91128 Palaiseau cedex, France

e-mail: stephane.gaubert@inria.fr

F. Meunier (凶)

Université Paris Est, LVMT, ENPC, 6-8 avenue Blaise Pascal, Cité Descartes Champs-sur-Marne, 77455 Marne-la-Vallée cedex 2, France

e-mail: frederic.meunier@enpc.fr 
Theorem 2 (Radon's theorem) Let $X$ be a set of $d+2$ points in $\mathbb{R}^{d}$. Then there are two pairwise disjoint subsets $X_{1}$ and $X_{2}$ of $X$ whose convex hulls have a common point.

Theorem 3 (Helly's theorem) Let $\mathcal{F}$ be a finite collection of convex sets in $\mathbb{R}^{d}$. If every $d+1$ members of $\mathcal{F}$ have a nonempty intersection, then the whole collection has a nonempty intersection.

Max-plus or tropical convexity arises when interpreting the notions of positive linear combination or of barycenter in the max-plus sense, meaning that the addition of scalars is replaced by the maximum and that the product of scalars is replaced by the addition.

This unusual convexity has been studied by several authors, with various motivations, including optimization (K. Zimmermann [31]), calculus of variations and asymptotic analysis (Litvinov, Maslov, Shpiz [18]), discrete event systems and optimal control (Cohen, Gaubert, Quadrat [9, 10]), tropical geometry (Develin and Sturmfels [11], whose work was the source of several developments, including [16] by Joswig, Sturmfels and Yu), and abstract convexity (Briec and Horvath [4], Nitica and Singer [22]).

Max-plus convexity is of special interest because max-plus objects arise as limits of a deformation in which classical algebraic objects are looked at with logarithmic glasses [30]. This deformation, which has been called "dequantization" by Maslov, by analogy with quasi-classics asymptotics, is at the origin of the current flourishing of tropical methods in algebraic geometry after the work of Viro [30] and Mikhalkin [21].

Several authors have obtained max-plus analogues of the previously mentioned discrete convexity theorems. The analogue of the Carathéodory theorem is mentioned by Helbig [15] and Develin and Sturmfels [11]. The analogue of Radon's theorem can be derived from results on max-plus linear independence by Gondran and Minoux [12, 13] and M. Plus [23] (see also [3, Sect. 3.4]), such a derivation was given by Butkovič [7] in the special case of vectors with finite entries and in [1] or [2] in the general case. The max-plus Radon theorem can also be obtained from the classical Radon theorem by a deformation argument, as in the work of Briec and Horvath [4] who also established the max-plus analogue of Helly's theorem using the same deformation idea. Gaubert and Sergeev [14] derived the max-plus Helly from a max-plus analogue of the theory of cyclic projections, leading to a direct combinatorial proof.

Other theorems have followed the ones of Carathéodory, Helly and Radon. In this paper, we show that these theorems also have max-plus versions.

Section 2 is devoted to the max-plus version of the beautiful colorful Carathéodory theorem proved by Bárány [8].

Theorem 4 (Colorful Carathéodory's theorem [8]) Suppose given $d+1$ finite point sets $X_{1}, X_{2}, \ldots, X_{d+1}$ and a point $\boldsymbol{p}$ in $\mathbb{R}^{d}$ such that the convex hull of each $X_{i}$ contains $\boldsymbol{p}$, then there are $d+1$ points $\boldsymbol{x}_{1}, \boldsymbol{x}_{2}, \ldots, \boldsymbol{x}_{d+1}$ such that $\boldsymbol{x}_{i} \in X_{i}$ for each $i$ and such that the point $\boldsymbol{p}$ is the convex hull of the points $\boldsymbol{x}_{1}, \boldsymbol{x}_{2}, \ldots, \boldsymbol{x}_{d+1}$. 
In Sect. 3, we briefly survey the existing approaches to the max-plus Radon theorem, and we point out that the max-plus Helly theorem can be derived from it, as in the case of classical convexity.

Radon's theorem has a beautiful generalization, Tverberg's theorem. The maxplus version is proved in Sect. 4.

Theorem 5 (Tverberg's theorem [28]) Let $X$ be a set of $(d+1)(q-1)+1$ points in $\mathbb{R}^{d}$. Then there are $q$ pairwise disjoint subsets $X_{1}, X_{2}, \ldots, X_{q}$ of $X$ whose convex hulls have a common point.

The case $q=2$ reduces to the usual Radon theorem.

A natural question is about the number of these partitions into $q$ subsets (each of these partition is called a Tverberg partition). A famous conjecture is the following one, also called the Dutch cheese conjecture, since Sierksma has offered a Dutch cheese for a solution of this problem.

Conjecture (Sierksma's conjecture) Let $q \geq 2, d \geq 1$ and put $N=(d+1)(q-1)$. For every $N+1$ points in $\mathbb{R}^{d}$, the number of unordered Tverberg partitions is at least $((q-1) !)^{d}$.

This conjecture is still open. One can naturally ask whether this conjecture holds in the max-plus setting. Surprisingly, it is possible to prove it in this case, with a quite simple proof. This is done in the last section of the paper.

Notation Before starting, we introduce some notation. We denote by $\mathbb{R}_{\max }$ the maxplus semiring, which consists of the elements of $\mathbb{R} \cup\{-\infty\}$. Capital letters will represent sets or collections, bold symbols (like $\boldsymbol{x}$ ) will represent vectors or points of an affine space, whereas scalars will be denoted with the usual typography. The set $\{1, \ldots, n\}$ will be denoted by $[n]$.

If $\lambda$ is in $\mathbb{R}_{\max }$ and if $\boldsymbol{x}$ denotes the vector $\left(x_{1}, \ldots, x_{d}\right)$, then, $\lambda+\boldsymbol{x}$ denotes the vector $\left(\lambda+x_{1}, \ldots, \lambda+x_{d}\right)$.

The usual convex hull of the points $x_{1}, \ldots, x_{n}$ in $\mathbb{R}^{d}$ is denoted by $\operatorname{conv}\left\{\boldsymbol{x}_{1}, \ldots, \boldsymbol{x}_{n}\right\}$ and the max-plus convex hull of the points $\boldsymbol{x}_{1}, \ldots, \boldsymbol{x}_{n}$ in $\mathbb{R}_{\max }^{d}$ by mpconv $\left\{\boldsymbol{x}_{1}, \ldots, \boldsymbol{x}_{n}\right\}$. The latter set consists of the max-plus convex combinations of the points $\boldsymbol{x}_{1}, \ldots, \boldsymbol{x}_{n}$. Each of these combinations is of the form $\max _{i \in[n]}\left(\lambda_{i}+\boldsymbol{x}_{i}\right)$, for some scalars $\lambda_{1}, \ldots, \lambda_{n}$ in $\mathbb{R}_{\max }$ such that $\max _{i \in[n]} \lambda_{i}=0$. Note that the former maximum which applies to the vectors $\lambda_{i}+\boldsymbol{x}_{i}$ is understood entrywise (for each of the $d$ components, one takes the maximum of the $n$ possible distinct values). The max-plus conic hull is defined in a way similar to the convex hull, but the requirement that $\max _{i \in[n]} \lambda_{i}=0$ is omitted.

\section{The Colorful Carathéodory Theorems}

Before considering the max-plus analogues of the colorful Carathéodory theorem, we restate the classical result in a slightly generalized form, straightforwardly derived from the classical one. 
Theorem 6 Suppose given $d+1$ finite point sets $X_{1}, X_{2}, \ldots, X_{d+1}$ and a convex set $C$ in $\mathbb{R}^{d}$ such that the convex hull of each $X_{i}$ intersects $C$. Then there are $d+1$ points $\boldsymbol{x}_{1}, \boldsymbol{x}_{2}, \ldots, \boldsymbol{x}_{d+1}$ such that $\boldsymbol{x}_{i} \in X_{i}$ for each $i$ and such that $\operatorname{conv}\left\{\boldsymbol{x}_{1}, \boldsymbol{x}_{2}, \ldots, \boldsymbol{x}_{d+1}\right\}$ intersects the convex $C$.

Proof For $i \in[d+1]$, one has $c_{i} \in C$ such that $c_{i} \in \operatorname{conv}\left(X_{i}\right)$. Then, the origin (zero vector) 0 belongs to the convex hull of each $X_{i}^{\prime}:=\left\{\boldsymbol{x}-\boldsymbol{c}_{i}: \boldsymbol{x} \in X_{i}\right\}$. Applying Theorem 4 to the sets $X_{1}^{\prime}, \ldots, X_{d+1}^{\prime}$ and the point $\boldsymbol{p}:=\mathbf{0}$ leads to the existence of $d+1$ points $\boldsymbol{x}_{1}, \boldsymbol{x}_{2}, \ldots, \boldsymbol{x}_{d+1}$ such that $\boldsymbol{x}_{i} \in X_{i}$ for each $i$ and such that $\mathbf{0} \in \operatorname{conv}\left\{\boldsymbol{x}_{1}-\right.$ $\left.c_{1}, \ldots, x_{d+1}-c_{d+1}\right\}$. Hence, $\operatorname{conv}\left\{c_{1}, \ldots, c_{d+1}\right\} \cap \operatorname{conv}\left\{x_{1}, \ldots, x_{d+1}\right\} \neq \emptyset$, and so $\operatorname{conv}\left\{\boldsymbol{x}_{1}, \boldsymbol{x}_{2}, \ldots, \boldsymbol{x}_{d+1}\right\}$ intersects the convex $C$.

Although the max-plus analogue of the classical form of the colorful Carathéodory theorem has a very simple proof, the generalized version with the convex set $C$ instead of the point $\boldsymbol{p}$ will need more advanced tools. Indeed, the reduction in the latter proof does not carry over to the max-plus case because the difference of vectors is no longer meaningful.

We first consider the max-plus analogue of Theorem 4.

Theorem 7 (Max-plus colorful Carathéodory's theorem, weak form) Suppose given $d+1$ finite point sets $X_{1}, X_{2}, \ldots, X_{d+1}$ and a point $\boldsymbol{p}$ in $\mathbb{R}_{\max }^{d}$ such that the max-plus convex hull of each $X_{i}$ contains $\boldsymbol{p}$. Then there are $d+1$ points $\boldsymbol{x}_{1}, \boldsymbol{x}_{2}, \ldots, \boldsymbol{x}_{d+1}$ such that $\boldsymbol{x}_{i} \in X_{i}$ for each $i$ and such that mpconv $\left\{\boldsymbol{x}_{1}, \boldsymbol{x}_{2}, \ldots, \boldsymbol{x}_{d+1}\right\}$ contains the point $\boldsymbol{p}$.

Proof The point $\boldsymbol{p}=\left(p_{1}, \ldots, p_{d}\right)$ can be written as

$$
\boldsymbol{p}=\max _{j \in[d+1]}\left(\lambda_{j}+\boldsymbol{y}^{(j)}\right),
$$

where $\lambda_{1}, \ldots, \lambda_{d+1} \in \mathbb{R}_{\max }, \max _{j \in[d+1]} \lambda_{j}=0$, and $\boldsymbol{y}^{(1)}, \ldots, \boldsymbol{y}^{(d+1)} \in X_{1}$. Reading the former equality only for the first component, we find an index $j$ such that the first component of $\lambda_{j}+\boldsymbol{y}^{(j)}$ is equal to $p_{1}$. Define $\boldsymbol{x}_{1}$ to be this $\boldsymbol{y}^{(j)}$ and $\mu_{1}$ to be the corresponding $\lambda_{j}$. Note that one has $\mu_{1}+\boldsymbol{x}_{1} \leq \boldsymbol{p}$ (componentwise), with equality for the first component.

The same argument allows us to find, for every $i \geq 2$ up to $i=d$, a scalar $\mu_{i}$ and a vector $\boldsymbol{x}_{i} \in X_{i}$ in such a way that $\mu_{i}+\boldsymbol{x}_{i} \leq \boldsymbol{p}$, with equality for the $i$ th component. Finally, we write again a decomposition of the form (1) in which every vector $\boldsymbol{y}^{(j)}$ belongs to $X_{d+1}$, but this time we choose $\boldsymbol{x}_{d+1} \in X_{d+1}$ to be a vector $\boldsymbol{y}^{(j)}$ such that $\lambda_{j}=0$ and we set $\mu_{d+1}:=0$. We still have $\mu_{d+1}+\boldsymbol{x}_{d+1} \leq \boldsymbol{p}$.

Thus, we constructed $d+1$ points $\boldsymbol{x}_{i} \in X_{i}$ for $i \in[d+1]$ such that $\max _{i \in[d+1]} \mu_{i}=0$ and

$$
\boldsymbol{p}=\max _{i \in[d+1]}\left(\mu_{i}+\boldsymbol{x}_{i}\right) .
$$

Theorem 8 (Max-plus colorful Carathéodory's theorem, strong form) Suppose given $d+1$ finite point sets $X_{1}, X_{2}, \ldots, X_{d+1}$ and a max-plus convex set $C$ in $\mathbb{R}_{\max }^{d}$ such that the max-plus convex hull of each $X_{i}$ intersects $C$. Then there 


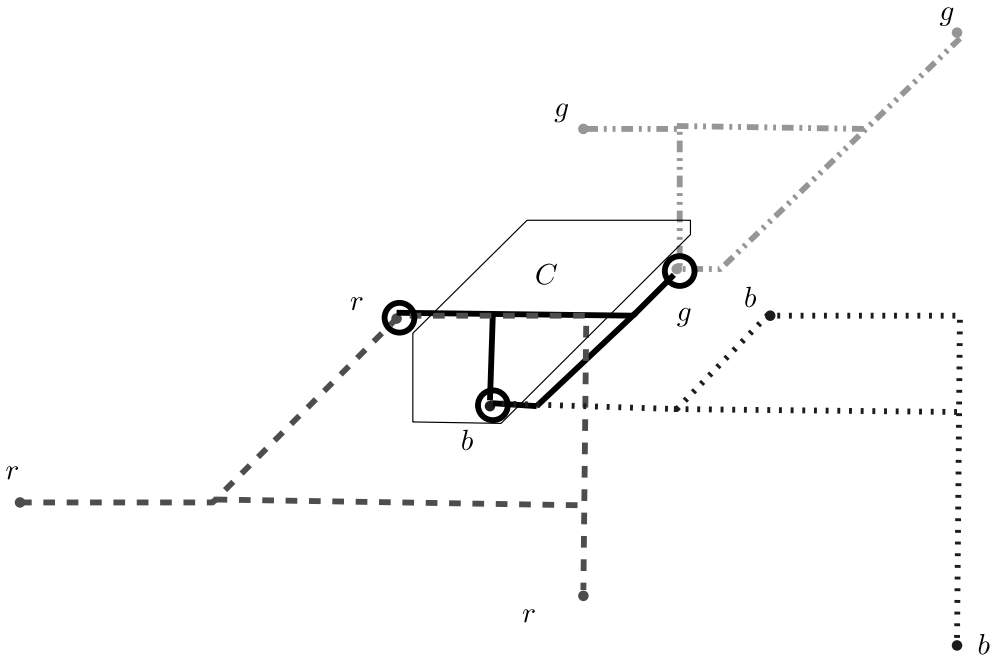

Fig. 1 Illustration of the max-plus generalized colorful Carathéodory theorem in dimension 2. There are three point sets: $X_{r}$ whose points are labeled with $r, X_{g}$ whose points are labeled with $g$, and $X_{b}$ whose points are labeled with $b$. The bold segments represent the "edges" of the max-plus "triangle" meeting $C$ and having a multicolor set of "vertices" (circles)

are $d+1$ points $\boldsymbol{x}_{1}, \boldsymbol{x}_{2}, \ldots, \boldsymbol{x}_{d+1}$ such that $\boldsymbol{x}_{i} \in X_{i}$ for each $i$ and such that $\operatorname{mpconv}\left\{\boldsymbol{x}_{1}, \boldsymbol{x}_{2}, \ldots, \boldsymbol{x}_{d+1}\right\}$ intersects the max-plus convex set $C$.

Figure 1 is an illustration of this theorem.

To prove this theorem, we shall make use of the following lemma:

Lemma 1 Consider an $n \times m$ matrix $A=\left(a_{i, j}\right)$ with entries in $\mathbb{R}_{\max }$. If $m \geq n$, then, for each column $i$ of $A$, it is possible to choose $\lambda_{j} \in \mathbb{R}_{\max }$ and add it to each entry of this column so that the $n$ row maxima of the new matrix are attained at $n$ positions belonging to different columns. Moreover, one can satisfy the additional requirement $\max _{j \in[m]} \lambda_{j}=0$.

Proof The proof works by induction on $n$. If $n=1$, there is nothing to prove. Hence suppose that $n>1$. Consider the bipartite graph $G$ whose color classes are $W:=$ $[n]$ (the rows of the matrix $A$ ) and $U:=[m]$ (the columns of the matrix $A$ ) and whose edges are those couples $(i, j)$ such that $a_{i, j} \neq-\infty$. Define the weight of the corresponding edge to be the real number $a_{i, j}$. Let us first assume that $G$ has at least one matching of cardinality $n$, which we may assume to match $W$ with the set $[n]$ of the first $n$ vertices of $U$. Let us consider the problem of finding a maximal weight perfect matching between these two sets of vertices. The dual linear programming problem reads

$$
\min \sum_{i \in[n]} u_{i}+\sum_{j \in[n]} v_{j}, \quad \boldsymbol{u}=\left(u_{i}\right), \boldsymbol{v}=\left(v_{j}\right) \in \mathbb{R}^{n}, u_{i}+v_{j} \geq a_{i j}, i, j \in[n]
$$


The duality theorem in linear programming shows that this problem has an optimal solution, $\boldsymbol{u}, \boldsymbol{v}$, which, by complementary slackness, is such that $u_{i}+v_{j}=a_{i j}$ for every edge $(i, j)$ belonging to a perfect matching of maximal weight. Moreover, since adding the same constant to the entries of $\boldsymbol{u}$ and subtracting it from the entries of $\boldsymbol{v}$ does not affect the optimality of $\boldsymbol{u}, \boldsymbol{v}$, we may assume that $\min _{j \in[n]} v_{j}=0$. Then, the weights $\lambda_{j}:=-v_{j}$ for $j \in[n]$ and $\lambda_{j}:=-\infty$ for $j>n$ have the desired properties.

Let us finally assume that there is no matching of cardinality $n$. By Hall's marriage theorem, there is a subset $X$ of $U$ whose neighborhood $N(X)$ in $G$ is such that $|N(X)|<|X| \leq n$. Applying the induction hypothesis to the matrix $A$ restricted to the columns indexed by $X$ and to the rows indexed by $N(X)$, we obtain the values of $\lambda_{j}$ for $j \in X$. We define $\lambda_{j}$ to be $-\infty$ on the other columns of $A$. Then, we easily check the required properties.

Proof of Theorem 8 For each $i$, we choose a point $\boldsymbol{b}^{(i)}=\left(b_{h}^{(i)}\right)$ in $C \cap \operatorname{mpconv}\left(X_{i}\right)$. Thinking of $\boldsymbol{b}^{(i)}$ as a column vector, we set

$$
\overline{\boldsymbol{b}}^{(i)}:=\left(\begin{array}{c}
\boldsymbol{b}^{(i)} \\
0
\end{array}\right)
$$

and define $A$ to be the $(d+1) \times(d+1)$ matrix $\left(\overline{\boldsymbol{b}}^{(1)} \ldots \overline{\boldsymbol{b}}^{(d+1)}\right)$.

Applying Lemma 1 to this matrix $A$, we get that there is a point $\boldsymbol{p}$ of $C$ such that

$$
\overline{\boldsymbol{p}}:=\left(\begin{array}{l}
\boldsymbol{p} \\
0
\end{array}\right)=\max _{i \in[d+1]}\left(\lambda_{i}+\overline{\boldsymbol{b}}^{(i)}\right)
$$

and such that each component is attained for a different $i$.

Now, for each $i$, as $\boldsymbol{b}^{(i)}$ is a max-plus convex combination of points in $X_{i}$, one has

$$
\overline{\boldsymbol{b}}^{(i)}=\max _{h \in[d+1]}\left(\mu_{h}^{(i)}+\overline{\boldsymbol{a}}_{h}^{(i)}\right), \quad \text { with } \boldsymbol{a}_{h}^{(i)} \in X_{i} \quad \text { and } \quad \overline{\boldsymbol{a}}_{h}^{(i)}:=\left(\begin{array}{c}
\boldsymbol{a}_{h}^{(i)} \\
0
\end{array}\right) \quad \text { for all } i, h .
$$

There is an index $i$ such that the first component of $\lambda_{i}+\overline{\boldsymbol{b}}^{(i)}$ is equal to the first one of $\overline{\boldsymbol{p}}$. Moreover, $\lambda_{i}+\overline{\boldsymbol{b}}^{(i)} \leq \overline{\boldsymbol{p}}$ (componentwise). Next, for this $i$, there is an $h(i) \in[d+1]$ such that $\mu_{h(i)}^{(i)}+\overline{\boldsymbol{a}}_{h(i)}^{(i)} \leq \overline{\boldsymbol{b}}^{(i)}$ with equality on the first component. Hence, one has $\lambda_{i}+\mu_{h(i)}^{(i)}+\overline{\boldsymbol{a}}_{h(i)}^{(i)} \leq \overline{\boldsymbol{p}}$ with equality on the first component.

We choose in the same way the index $h(i)$ for the different values of the column index $i$ arising by considering indices attaining the row maxima in $A$. These indices $i$ have been chosen to be all distinct a few lines above, and so

$$
\max _{i \in[d+1]}\left(\lambda_{i}+\mu_{h(i)}^{(i)}+\overline{\boldsymbol{a}}_{h(i)}^{(i)}\right)=\overline{\boldsymbol{p}} .
$$

This can be rewritten as

$$
\max _{i \in[d+1]}\left(\lambda_{i}+\mu_{h(i)}^{(i)}+\boldsymbol{a}_{h(i)}^{(i)}\right)=\boldsymbol{p},
$$


where $\boldsymbol{a}_{h(i)}^{(i)}$ is a point of $X_{i}$ for each $i \in[d+1]$ and $\max _{i \in[d+1]}\left(\lambda_{i}+\mu_{h(i)}^{(i)}\right)=0$. Define then $\boldsymbol{x}_{i}:=\boldsymbol{a}_{h(i)}^{(i)}$. The point $\boldsymbol{p}$ belongs to $C$ and is a max-plus convex combination of the points $\boldsymbol{x}_{i} \in X_{i}$, as required.

\section{Radon's and Helly's Theorems}

The max-plus Radon theorem is:

Theorem 9 (Max-plus Radon's theorem [1, 4, 7, p. 13]) Let $X$ be a set of $d+2$ points in $\mathbb{R}_{\max }^{d}$. Then there are two pairwise disjoint subsets $X_{1}$ and $X_{2}$ of $X$ whose max-plus convex hulls have a common point.

An illustration is given in Fig. 2.

We next briefly discuss different approaches to this result which have appeared in the literature or which can be derived from it. The first one, relying on a deformation argument, will give more insight on the proof of the max-plus Tverberg theorem that we give in the next section.

Consider the map $E_{\beta}$ sending a vector $\boldsymbol{x}=\left(x_{i}\right) \in \mathbb{R}_{\max }^{d}$ to the vector of $\mathbb{R}^{d}$ with coordinates $\exp \left(\beta x_{i}\right)$, where $\beta>0$ is a scaling parameter. We introduce, following Maslov [19], the addition $\boldsymbol{x}+\beta \boldsymbol{y}:=E_{\beta}^{-1}\left(E_{\beta}(\boldsymbol{x})+E_{\beta}(\boldsymbol{y})\right)$, for $\boldsymbol{x}, \boldsymbol{y} \in \mathbb{R}_{\max }^{d}$, which is such that $\boldsymbol{x}+\beta \boldsymbol{y}$ converges to $\max (\boldsymbol{x}, \boldsymbol{y})$ uniformly in $\boldsymbol{x}$ and $\boldsymbol{y}$ as $\beta \rightarrow \infty$. This suggests to define a $\beta$-convex combination of a set of vectors $X=\left\{\boldsymbol{x}_{1}, \ldots, \boldsymbol{x}_{n}\right\}$ to be an element of the form $\left(\mu_{1}+\boldsymbol{x}_{1}\right)+{ }_{\beta} \cdots+{ }_{\beta}\left(\mu_{n}+\boldsymbol{x}_{n}\right)$ where $\mu_{1}, \ldots, \mu_{n} \in \mathbb{R} \cup$ $\{-\infty\}$ are such that $\mu_{1}+\beta \cdots+\beta \mu_{n}=0$. The $\beta$-convex hull $\operatorname{co}_{\beta}(X)$ is defined as the set of all such combinations, it coincides with the image by the map $E_{\beta}^{-1}$ (the "logarithmic glasses") of the classical convex hull of the set $E_{\beta}(X)$. The same deformation is of fundamental importance in tropical geometry [21, 24, 30]. Briec and Horvath considered in [4] an equivalent deformation. Their result shows that the upper limit in the Painlevé-Kuratovski sense of $\operatorname{co}_{\beta}(X)$ as $\beta \rightarrow \infty$ is precisely the max-plus convex hull of the finite set $X$. Then, the max-plus Radon theorem follows readily from the classical one.
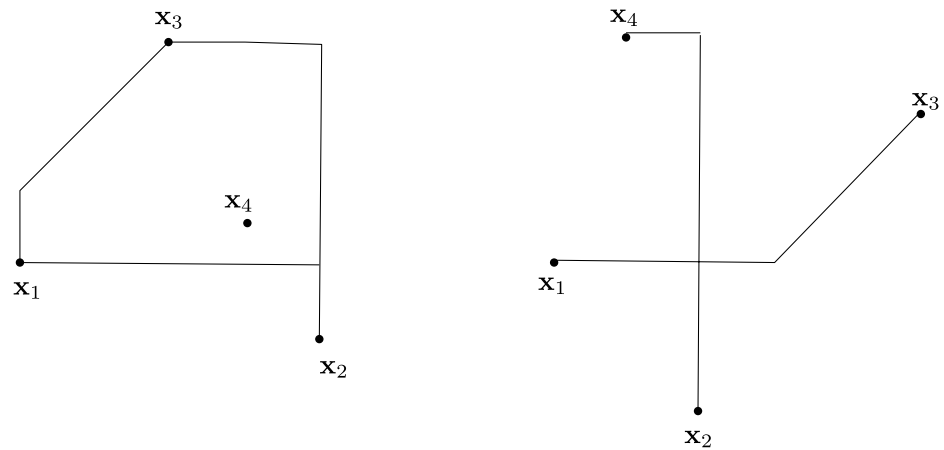

Fig. 2 Illustration of the max-plus Radon theorem in dimension 2 
An alternative approach, originating from the work of Gondran and Minoux $[12,13]$ and by M. Plus [23], gives a combinatorial information on the Radon's partitions. We shall consider the equivalent conic version of the result. Define the positive (max-plus) determinant of a $n \times n$ matrix $B=\left(b_{i j}\right)$ as the maximum of the sums $\sum_{i \in[n]} b_{i \sigma(i)}$ over all even permutations $\sigma$. The negative determinant is defined by taking the odd permutations instead of the even ones.

Combinatorial identities in rings are known to have semiring analogues when written "without minus sign", as was first observed by Reutenauer and Straubing [25], see also [1, 13, 23], and [2] were a formal "transfer principle" is stated. In particular, for every family $\boldsymbol{x}_{1}, \ldots, \boldsymbol{x}_{d+1}$ of vectors of dimension $d$ with entries in a commutative ring, the homogeneous form of the Cramer formula shows that $D_{1} \boldsymbol{x}_{1}-D_{2} \boldsymbol{x}_{2}+\cdots=0$ where the alternated sum has $d+1$ terms, and for all $i \in[d+1], D_{i}$ denotes the determinant of the matrix $X_{i}$ with columns $\boldsymbol{x}_{1}, \ldots, \boldsymbol{x}_{i-1}, \boldsymbol{x}_{i+1}, \ldots, \boldsymbol{x}_{d+1}$. Then, the following max-plus version of the latter combinatorial identity is valid:

$$
\max \left(D_{1}^{+}+\boldsymbol{x}_{1}, D_{2}^{-}+\boldsymbol{x}_{2}, \ldots\right)=\max \left(D_{1}^{-}+\boldsymbol{x}_{1}, D_{2}^{+}+\boldsymbol{x}_{2}, \ldots\right)
$$

where $D_{i}^{+}$(resp., $D_{i}^{-}$) denotes the positive (resp., negative) max-plus determinant of the matrix $X_{i}$, and each maximum comprises $d+1$ terms. For generic values of the entries of the vectors $x_{1}, \ldots, \boldsymbol{x}_{d+1}$, we have $D_{i}^{+} \neq D_{i}^{-}$for all $i$. Then, define $I^{+}$to be set consisting of the odd indices from which $D_{i}^{+}>D_{i}^{-}$and of the even indices for which $D_{i}^{-}>D_{i}^{+}$, define $I^{-}$in the symmetric way, and set $D_{i}=\max \left(D_{i}^{+}, D_{i}^{-}\right)$. Then, for every entry of the vector maximum at the left-hand side of (2), the maximum must necessarily be attained by some $i$ th term with $i \in I^{+}$. The same is true for the right-hand side, with $I^{-}$, and so

$$
\max _{i \in I^{+}}\left(D_{i}+\boldsymbol{x}_{i}\right)=\max _{i \in I^{-}}\left(D_{i}+\boldsymbol{x}_{i}\right)
$$

Thus $\left(I^{+}, I^{-}\right)$is a conic Radon partition for the family $\boldsymbol{x}_{1}, \ldots, \boldsymbol{x}_{d+1}$. The general case can be obtained from the generic one by an immediate density argument.

Identity (3) is intimately related to the two distinct max-plus or tropical analogues of the Cramer formula given by M. Plus [23] (a recent account can be found in [2], see also [3, §3.4]) and Richter-Gebert, Sturmfels, and Theobald [24]. It may also be derived from a result of Gondran and Minoux $[12,13]$ on max-plus linear independence, as shown by Butkovič [7] (under the minor restriction that the vectors $\boldsymbol{x}_{i}$ have finite entries) and by Akian, Bapat, and Gaubert [1, p. 13].

We note that computing the max-plus "Cramer permanent" $D_{i}$ is equivalent to solving an optimal assignment problem. Richter-Gebert, Sturmfels, and Theobald [24], following an earlier idea of Sturmfels and Zelevinsky [27], showed that all the max-plus Cramer permanents $D_{1}, \ldots, D_{d+1}$ can be computed simultaneously up to an additive constant, by solving a single transportation problem, under some non-degeneracy condition. We also note that checking whether $D_{i}^{+}=D_{i}^{-}$reduces to finding an elementary even cycle in a digraph, as shown by Butkovič [6].

We next point out that Helly's theorem can be straightforwardly derived from Radon theorem, as in the case of classical convexity. The max-plus version was first 
proved by Briec and Horvath in [4], by exploiting the deformation method above, and by Gaubert and Sergeev, as a consequence of their work on cyclic projections [14].

Theorem 10 (Max-plus Helly's theorem [4, 14]) Let $\mathcal{F}$ be a finite collection of maxplus convex sets in $\mathbb{R}_{\max }^{d}$. If every $d+1$ members of $\mathcal{F}$ have a nonempty intersection, then all the members of $\mathcal{F}$ have a nonempty intersection.

Proof Let $C_{1}, \ldots, C_{n}$ be $n$ max-plus convex sets in $\mathbb{R}_{\max }^{d}$ and suppose that whenever $d+1$ sets among them are selected, they have a nonempty intersection. The proof works by induction on $n$. We first assume that $n=d+2$. Define $\boldsymbol{x}_{i}$ to be a point in $\bigcap_{j=1, j \neq i}^{d+2} C_{j}$. We have then $d+2$ points $\boldsymbol{x}_{1}, \ldots, \boldsymbol{x}_{d+2}$. If two of them are equal, then this point is in the whole intersection. Hence, we can assume that all the $\boldsymbol{x}_{i}$ are different. By the max-plus Radon theorem, we have two disjoint subsets $S$ and $T$ partitioning $[d+2]$ such that there is a point $\boldsymbol{x}$ in mpconv $\left(\bigcup_{i \in S} \boldsymbol{x}_{i}\right) \cap \operatorname{mpconv}\left(\bigcup_{i \in T} \boldsymbol{x}_{i}\right)$. This point $\boldsymbol{x}$ belongs to every $C_{i}$.

Indeed, take $j \in[d+2]$, which is either in $S$ or in $T$. Suppose without loss of generality that $j$ is in $S$. Then, $\operatorname{mpconv}\left(\bigcup_{i \in T} \boldsymbol{x}_{i}\right)$ is included in $C_{j}$, and so $\boldsymbol{x} \in C_{j}$. The case $n=d+2$ is proved.

Suppose now that $n>d+2$ and that the theorem is proved up to $n-1$. Define $C_{n-1}^{\prime}:=C_{n-1} \cap C_{n}$. When $d+2$ max-plus convex sets $C_{i}$ are selected, they have a nonempty intersection, according to what we have just proved. Hence, every $d+1$ members of the collection $C_{1}, \ldots, C_{n-2}, C_{n-1}^{\prime}$ have a nonempty intersection. By induction, the whole collection has a nonempty intersection.

\section{Tverberg's Theorem}

We have a Tverberg theorem in the max-plus framework:

Theorem 11 (Max-plus Tverberg's theorem) Let $X$ be a set of $(d+1)(q-1)+1$ points in $\mathbb{R}_{\max }^{d}$. Then there are $q$ pairwise disjoint subsets $X_{1}, X_{2}, \ldots, X_{q}$ of $X$ whose max-plus convex hulls have a common point.

Figure 3 illustrates this theorem for $d=2, q=3$. The partition emphasized is $X_{1}=\left\{x_{1}, x_{5}\right\}, X_{2}=\left\{x_{2}, x_{4}, x_{7}\right\}$ and $X_{3}=\left\{x_{3}, x_{6}\right\}$.

To establish this theorem, we will combine the beautiful ideas introduced by Sarkaria [26] and streamlined by Bárány and Onn [5] and Matoušek [20] to prove the usual (non max-plus) Tverberg theorem, with a variant of the deformation technique discussed in the previous section.

Proof Put $N:=(d+1)(q-1)$. We shall only prove the conic version. Indeed, the convex version can be straightforwardly derived from it by adding to each point a $(d+1)$ th component equal to 0 . The conic version can be stated as follows: Let $X=\left\{\boldsymbol{a}_{1}, \ldots, \boldsymbol{a}_{N+1}\right\}$ be a set of $N+1$ points in $\mathbb{R}_{\max }^{d+1} \backslash\{(-\infty, \ldots,-\infty)\}$. Then there are $q$ pairwise disjoint subsets $X_{1}, X_{2}, \ldots, X_{q}$ of $X$ whose max-plus conic hulls have a common point different from $(-\infty, \ldots,-\infty)$. 


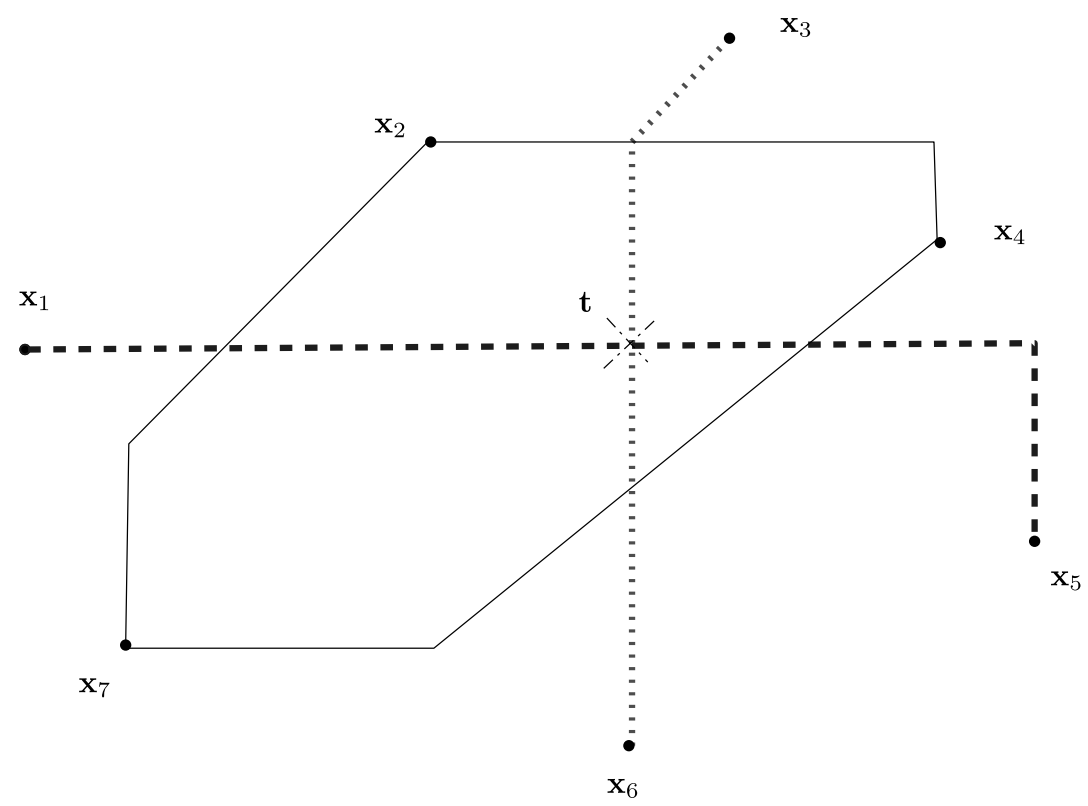

Fig. 3 Max-plus Tverberg theorem for $d=2$ and $q=3$

Define the linear maps $\phi_{j}: \mathbb{R}^{d+1} \rightarrow \mathbb{R}^{(d+1)(q-1)}$ for $j \in[q]$ by $\phi_{j}(\boldsymbol{y})=(\mathbf{0}, \ldots, \mathbf{0}, \boldsymbol{y}, \mathbf{0}, \ldots, \mathbf{0}) \in\left(\mathbb{R}^{d+1}\right)^{q-1}, \quad$ for $j<q, \mathbf{0} \in \mathbb{R}^{d+1}$ and $\boldsymbol{y} \in \mathbb{R}^{d+1}$ where $\boldsymbol{y}$ is in $j$ th position. Moreover, set $\phi_{q}(\boldsymbol{y})=(-\boldsymbol{y},-\boldsymbol{y}, \ldots,-\boldsymbol{y})$ for $\boldsymbol{y} \in \mathbb{R}^{d+1}$. For every $\boldsymbol{y} \in \mathbb{R}^{d+1}$, we have $\mathbf{0} \in \operatorname{conv}\left\{\phi_{1}(\boldsymbol{y}), \ldots, \phi_{q}(\boldsymbol{y})\right\}$.

Let $u$ denote a real positive parameter, and let us associate to the point $\boldsymbol{a}_{i}=$ $\left(a_{1, i}, \ldots, a_{d+1, i}\right)$ the point $\boldsymbol{\alpha}_{i}(u)=\left(u^{a_{1, i}}, \ldots, u^{a_{d+1, i}}\right) \in \mathbb{R}^{d+1}$, with the convention that $u^{-\infty}:=0$.

Suppose first that the parameter $u$ is fixed. We can apply the colorful Carathéodory theorem to the sets of points $\tilde{X}_{1}, \ldots, \tilde{X}_{N+1}$, where $\tilde{X}_{i}:=\left\{\phi_{1}\left(\boldsymbol{\alpha}_{i}(u)\right), \ldots, \phi_{q}\left(\boldsymbol{\alpha}_{i}(u)\right)\right\}$. Indeed, we have $\mathbf{0} \in \operatorname{conv}\left(\tilde{X}_{i}\right)$ for each $i \in[N+1]$ and the sets $\tilde{X}_{i}$ consist of points of $\mathbb{R}^{N}$. We deduce that there exist $j_{1}, j_{2}, \ldots, j_{N+1}$ in $[q]$ and real numbers $\mu_{1}, \ldots, \mu_{N+1}$ such that

$$
\mu_{i} \geq 0, \quad i \in[N+1] \quad \sum_{i \in[N+1]} \mu_{i}=1, \quad \mathbf{0}=\sum_{i \in[N+1]} \mu_{i} \phi_{j_{i}}\left(\boldsymbol{\alpha}_{i}(u)\right) .
$$

Of course, the coefficients $\mu_{i}$ depend on $u$, and so do the indices $j_{i}$.

Let us now make $u$ vary. More precisely, we assume that $u$ takes the values of a sequence $\left(u_{m}\right)$ of real positive numbers, indexed by $m \geq 1$, and such that $u_{m}$ tends to infinity. Since there is only a finite number of possible choices of the indices $j_{i}$, we may assume, possibly after replacing $\left(u_{m}\right)$ by a subsequence, that the relations (4) hold for all $u=u_{m}$, with $m \geq 1$. Since the set of $\mu_{i}$ satisfying these relations is a 
polyhedron included in $\mathbb{R}^{N+1}$, we may choose a solution $\boldsymbol{\mu}=\boldsymbol{\mu}(u)=\left(\mu_{i}(u)\right)$ to be a vertex of this polyhedron, whose entries are of the form

$$
\mu_{i}(u)=c_{i}(u) / b(u), \quad i \in[N+1]
$$

where $c_{i}(u)$ and $b(u)$ are given by polynomials expressions with real coefficients and real exponents in the parameter $u$. In other words, $c_{i}(u)$ and $b(u)$ can be represented as finite sums

$$
\sum_{k} \gamma_{k} u^{\beta_{k}}, \quad \text { with } \gamma_{k}, \beta_{k} \in \mathbb{R}
$$

Indeed, the denominator $b(u)$ in (5) can be chosen to be the determinant of some invertible submatrix arising from the system of equalities saturated by the extreme point $\boldsymbol{\mu}(u)$, and then a polynomial expression for $c_{i}(u)$ is straightforwardly derived. Again, we may assume that the submatrix defining the extreme point is the same for all $u=u_{m}$, so that (4) and (5) hold for all $u=u_{m}$ with $m \geq 1$.

Define $S_{l}$ to be the set of indices $i \in[N+1]$ such that $j_{i}=l$, so that the sets $S_{1}, \ldots, S_{q}$ are pairwise distinct (and non-empty since we have $\sum_{i \in[N+1]} \mu_{i}(u)=1$ ). Using the definition of the maps $\phi_{j}$, we deduce from the last equation in (4) that the following holds as $u=u_{m}$ tends to infinity:

$$
\sum_{i \in S_{1}} \mu_{i}(u)\left(\begin{array}{c}
u^{a_{1, i}} \\
\vdots \\
u^{a_{d+1, i}}
\end{array}\right)=\cdots=\sum_{i \in S_{q}} \mu_{i}(u)\left(\begin{array}{c}
u^{a_{1, i}} \\
\vdots \\
u^{a_{d+1, i}}
\end{array}\right) .
$$

We define the leading exponent of the expression (6) to be the number $\sup \left\{\beta_{k} \mid \gamma_{k} \neq 0\right\}$, with the convention that the leading exponent of the zero expression is $-\infty$. Clearly, two polynomial expressions of $u$ which coincide as $u=u_{m}$ tends to infinity must have the same leading exponent. Let $\lambda_{i}$ denote the leading exponent of $c_{i}(u)$. We deduce that

$$
\max _{i \in S_{1}}\left(\lambda_{i}+\boldsymbol{a}_{i}\right)=\cdots=\max _{i \in S_{q}}\left(\lambda_{i}+\boldsymbol{a}_{i}\right) .
$$

We also deduce from $\sum_{i \in[N+1]} \mu_{i}(u)=1$ that the $\lambda_{i}$ are not all equal to $-\infty$. Since by assumption each $\boldsymbol{a}_{i}$ has at least one component $\neq-\infty$, the point appearing in all the members of $(8)$ is different from $(-\infty, \ldots,-\infty)$. The conclusion follows.

\section{Dutch Cheese Conjecture}

We finish the article with the max-plus version of Sierksma's conjecture, which turns out to be a theorem.

Theorem 12 Let $q \geq 2, d \geq 1$ and put $N=(d+1)(q-1)$. For every $N+1$ points in $\mathbb{R}_{\max }^{d}$ the number of unordered max-plus Tverberg partitions is at least $((q-1) !)^{d}$. 
For instance, if $d=2$ and $q=3$, this theorem says that we have at least 4 partitions. We can check this assertion in the particular case given in Fig. 3. One partition is emphasized. There must be three other ones. Indeed,

$$
\begin{array}{lll}
X_{1}=\left\{x_{1}, x_{5}\right\}, & X_{2}=\left\{x_{2}, x_{6}\right\}, & X_{3}=\left\{x_{3}, x_{4}, x_{7}\right\}, \\
X_{1}=\left\{x_{1}, x_{4}\right\}, & X_{2}=\left\{x_{3}, x_{6}\right\}, & X_{3}=\left\{x_{2}, x_{5}, x_{7}\right\}, \\
X_{1}=\left\{x_{1}, x_{4}\right\}, & X_{2}=\left\{x_{2}, x_{6}\right\}, & X_{3}=\left\{x_{3}, x_{5}, x_{7}\right\}
\end{array}
$$

are three other Tverberg partitions.

To prove this theorem, we will use a purely combinatorial result-Corollary 1 below-concerning a partition of a color class of a bipartite graph. It has in a sense a "Tverberg" nature. To prove this result, it is useful to prove the following theorem.

For a graph $G=(V, E)$, recall that $N(X)$ denotes the neighborhood of $X$, that is the set of vertices in $V \backslash X$ having at least one neighbor in $X$.

Theorem 13 Let $G$ be a bipartite graph with color classes $U$ and $W$ and no isolated vertices, and let $q$ be a positive integer. If $|U| \geq(q-1)|W|+1$, then there are $q$ disjoint subsets $U_{1}, \ldots, U_{q}$ of $U$ such that $N\left(U_{1}\right)=N\left(U_{2}\right)=\cdots=N\left(U_{q}\right)$. Moreover, there are at least $((q-1) !)^{\left|N\left(U_{1}\right)\right|-1}$ distinct ways of choosing these $q$ subsets (one does not take the order into account).

The first part of this theorem, i.e., the existence of the $q$ subsets, was already proved by Lindström [17] and by Tverberg with another proof [29]. The bound on the number of ways of choosing these $q$ subsets is new.

Corollary 1 Let $G=(V, E)$ be a bipartite graph whose color classes are $U$ and $W$. Suppose that for all $Y \subseteq W, Y \neq \emptyset$, one has

$$
|N(Y)| \geq(q-1)|Y|+1 .
$$

Then $U$ can be partitioned into $q$ subsets $U_{1}, \ldots, U_{q}$ such that for all $i \in[q]$ one has $N\left(U_{i}\right)=W$. Moreover, there are at least $((q-1) !)^{|W|-1}$ distinct partitions satisfying this property (one does not take the order into account).

Proof of Theorem 13 The proof works by induction on $|U|$. If $|U|=q$, the theorem is clearly true. Hence, let $|U| \geq q+1$. We can assume that for all $X \subsetneq U$, we have $|X| \leq$ $(q-1)|N(X)|$ (if not, apply induction). We will prove that there exists a partition $U_{1}, \ldots, U_{q} \subseteq U$ such that $N\left(U_{i}\right)=W$ for all $i=1, \ldots, q$, and explain why in this case $((q-1) !)^{|W|-1}$ is a lower bound of the distinct ways of choosing these $q$ subsets, when we do not take the order into account.

Choose a subset $U^{\prime} \subseteq U$ of cardinality $(q-1)|W|$. We can apply Hall's marriage theorem and get a subset of edges $F \subseteq E$ such that $\operatorname{deg}_{F}(w)=q-1$ for each $w \in W$ and $\operatorname{deg}_{F}(u)=1$ for each $u \in U^{\prime}$ (make $q-1$ copies of each vertex $w$ of $W$ to see it). Note that there is no subset $A \neq \emptyset$ of $W$ such that $|N(A)| \leq(q-1)|A|$, otherwise we would have a subset $X:=U \backslash N(A) \subseteq U$ such that $(q-1)|N(X)|<|X|$ since 
$N(X) \subseteq W \backslash A$. Hence, it is possible to find an order $w_{1}, \ldots, w_{|W|}$ of the vertices of $W$ such that

$$
N\left(w_{i}\right) \cap\left(Y_{1} \cup \cdots \cup Y_{i-1} \cup\left(U \backslash U^{\prime}\right)\right) \neq \emptyset \quad \text { for all } i=1, \ldots,|W|,
$$

where $Y_{j}$ denotes the neighbors of $w_{j}$ in $F$. In the case when $i=1$, we require simply that $N\left(w_{1}\right) \cap\left(U \backslash U^{\prime}\right) \neq \emptyset$.

We define now the $U_{i}$ in order to have $N\left(U_{i}\right)=W$ for $i=1, \ldots, q$ by adding vertices. Start with $U_{1}=\cdots=U_{q}=\emptyset$.

Add $U \backslash U^{\prime}$ to $U_{1}$. Put the $q-1$ vertices of $Y_{1}$ respectively in $U_{2}, \ldots, U_{q}$. The vertex $w_{1}$ is now in the neighborhood of $U_{1}, \ldots, U_{q}$. Process $w_{2}, \ldots, w_{|W|}$ in this order. The processing of $w_{i}$ consists first in finding the index $j^{*}$ such that $w_{i}$ is already in $N\left(U_{j^{*}}\right)$. Such a $j^{*}$ exists because of property (**). Second, it consists in adding to each of the $U_{j}$, except for $j=j^{*}$, one of the $q-1$ vertices of $Y_{i}$. This ensures that when the processing of $w_{i}$ is finished, $w_{i}$ is in the neighborhood of $U_{1}, \ldots, U_{q}$. Since all vertices of $W$ are eventually processed, we get $N\left(U_{1}\right)=\cdots=$ $N\left(U_{q}\right)=W$.

It is easy to see why the lower bound on the number of ways of choosing these $q$ subsets is true. Indeed, there are $q$ ! ways of processing the vertex $w_{1}$ : The subset $U \backslash U^{\prime}$ can be added to any of the subsets $U_{j}$ and the vertices of $Y_{1}$ to the $q-1$ other subsets $U_{j}$ in any order. For each of the vertices $w_{i}$, there are $(q-1)$ ! ways for adding the vertices of $Y_{i}$ to each of the remaining $U_{j}$. We get $q !((q-1) !)^{|W|-1}$ different ways. Since we do not take the order into account, we have the required lower bound.

Proof of Corollary 1 Apply Theorem 13. We get $q$ disjoint subsets $U_{1}^{(1)}, \ldots, U_{q}^{(1)}$ having the same neighborhood in $W$. Define $U^{\prime}:=U \backslash \bigcup_{i=1}^{q} U_{i}^{(1)}$ and $W^{\prime}:=W \backslash$ $N\left(U_{1}^{(1)}\right)$. For all $Y \subseteq W^{\prime}$, we have $N(Y) \subseteq U^{\prime}$. Hence we can apply Theorem 13 on the subgraph induced by $U^{\prime} \cup W^{\prime}$, and get $U_{1}^{(2)}, \ldots, U_{q}^{(2)}$ disjoint subsets of $U^{\prime}$ having the same neighborhood in $W^{\prime}$. And so on. At the end, just define $U_{i}$ to be the union of all $U_{i}^{(j)}$ defined through this process, for each $i=1, \ldots, q$.

The lower bound for the number of distinct partitions is easily derived.

We will soon prove Theorem 12. In the proof, we will need to show that we have a condition that translates into condition $(*)$ of Corollary 1 . This is done by the following lemma:

Lemma 2 For a set $X=\left\{\boldsymbol{x}_{1}, \boldsymbol{x}_{2}, \ldots, \boldsymbol{x}_{n}\right\}$ of generic points in $\mathbb{R}_{\max }^{d+1}$, if $n \leq(d+1) \times$ $(q-1)$, then there is no max-plus conic Tverberg partition into $q$ disjoint subsets.

Proof The proof works by contradiction. Suppose that we have a max-plus conic Tverberg partition $X_{1}, \ldots, X_{q}$. Define $\boldsymbol{x}$ to be a Tverberg point, that is a point in the common intersections of the conic hulls of the $X_{i}$. Define moreover $\lambda_{i}$ to be the coefficient of $\boldsymbol{x}_{i}$ in the Tverberg partition.

Consider the graph $H=(V, E)$ where $V:=X$ and there is an edge between $\boldsymbol{x}_{i}$ and $\boldsymbol{x}_{j}$ if the following two conditions are satisfied: (i) $\boldsymbol{x}_{i}$ and $\boldsymbol{x}_{j}$ are in two consecutive subsets, that is, there is an $l \in\{1, \ldots, q\}$ such that $\boldsymbol{x}_{i} \in X_{l}$ and $\boldsymbol{x}_{j} \in X_{l+1}$, 
and (ii) $\lambda_{i}+\boldsymbol{x}_{i}$ and $\lambda_{j}+\boldsymbol{x}_{j}$ coincide in at least one coordinate. Parallel edges are allowed.

For each coordinate, $H$ gets at least $q-1$ edges. Hence $H$ has at least $(d+1)(q-1) \geq n$ edges, and thus has at least one cycle $C$. Without loss of generality, let $C:=\left(\boldsymbol{x}_{1}, \ldots, \boldsymbol{x}_{c}\right)$ in this order, where $c$ is the size of the cycle. We write $\boldsymbol{x}_{i}:=\left(x_{1, i}, \ldots, x_{d+1, i}\right)$, and define $j(i)$ the coordinate such that $\lambda_{i}+x_{i, j(i)}=$ $\lambda_{i+1}+x_{i+1, j(i)}$. Summing the left- and right-hand sides of the equality leads to the following equality:

$$
\sum_{i \in C} x_{i, j(i)}=\sum_{i \in C} x_{i+1, j(i)} .
$$

Since $j(1), j(2), \ldots, j(c)$ are not all equal, otherwise the edges provided by a coordinate would span a cycle (which would contradict condition (i) defining $H$ ), one of the terms on the left-hand side of the equality does not appear on the right-hand side. But then the equality is in contradiction with the genericity assumption.

Proof of Theorem 12 We work with the conic version, for there is a one-to-one correspondence between the max-plus Tverberg partitions in the conic and the convex settings.

Let us start with a particular max-plus Tverberg partition, the existence of which follows from Theorem 11. We have $X_{1}, \ldots, X_{q}$ that provide a partition of $X=$ $\left\{\boldsymbol{x}_{1}, \ldots, \boldsymbol{x}_{N+1}\right\}$. Recall that the points $\boldsymbol{x}_{i}$ belong to $\mathbb{R}_{\max }^{d+1}$. Define $\boldsymbol{x}$ to be a Tverberg point, that is, a point in the common intersections of the conic hulls of the $X_{i}$ that is different from $(-\infty, \ldots,-\infty)$. Define moreover $\lambda_{i}$ to be the coefficient of $\boldsymbol{x}_{i}$ in the Tverberg partition.

Consider the following bipartite graph with color classes $U:=X$ and $W:=$ $[d+1]$. We put an edge between $\boldsymbol{x}_{i} \in X$ and $j \in[d+1]$ if the $j$ th components of $\lambda_{i}+\boldsymbol{x}_{i}$ and $\boldsymbol{x}$ coincide. Our max-plus Tverberg partition $X_{1}, \ldots, X_{q}$ provides a partition of $U$ into subsets $U_{1}, \ldots, U_{q}$ such that for all $i$ we have $N\left(U_{i}\right)=W$ by putting $U_{i}:=X_{i}$. Moreover, by a slight perturbation, according to Lemma 2, we get that we need at least $\left(d^{\prime}+1\right)(q-1)+1$ points to have a conic Tverberg partition in dimension $d^{\prime}+1$, for any $0 \leq d^{\prime} \leq d$. Hence, our bipartite graph satisfies all conditions of Corollary 1.

Now remark that each partition of $U$ into subsets $U_{1}, \ldots, U_{q}$ such that $N\left(U_{i}\right)=W$ for each $i=1, \ldots, q$ provides a max-plus Tverberg partition by putting $X_{i}:=U_{i}$. Corollary 1 implies thus the required lower bound for the number of max-plus Tverberg partitions.

Acknowledgements We thank Helge Tverberg for having pointed out the references [17, 29].

\section{References}

1. Akian, M., Bapat, R., Gaubert, S.: Max-plus algebra. In: Hogben, L., Brualdi, R., Greenbaum, A., Mathias, R. (eds.) Handbook of Linear Algebra. Chapman and Hall, London (2006)

2. Akian, M., Gaubert, S., Guterman, A.: Linear independence over tropical semirings and beyond. In: Litvinov, G.L., Sergeev, S.N. (eds.) The Proceedings of the International Conference on Tropical and Idempotent Mathematics. Contemp. Math. AMS (2008, to appear). arXiv:0812.3496 
3. Baccelli, F., Cohen, G., Olsder, G.J., Quadrat, J.P.: Synchronization and Linearity. Wiley, New York (1992)

4. Briec, W., Horvath, C.: $\mathbb{B}$-convexity. Optimization 53, 103-127 (2004)

5. Bárány, I., Onn, S.: Colourful linear programming and its relatives. Math. Oper. Res. 22, 550-567 (1997)

6. Butkovič, P.: Regularity of matrices in min-algebra and its time-complexity. Discrete Appl. Math. 57(2-3), 121-132 (1995). Combinatorial optimization 1992 (CO92) (Oxford)

7. Butkovič, P.: Max-algebra: the linear algebra of combinatorics? Linear Algebra Appl. 367, 313-335 (2003)

8. Bárány, I.: A generalization of Carathéodory's theorem. Discrete Math. 40, 141-152 (1982)

9. Cohen, G., Gaubert, S., Quadrat, J.P.: Hahn-Banach separation theorem for max-plus semimodules. In: Menaldi, J.L., Rofman, E., Sulem, A. (eds.) Optimal Control and Partial Differential Equations, pp. 325-334. IOS Press, Amsterdam (2001)

10. Cohen, G., Gaubert, S., Quadrat, J.-P.: Duality and separation theorems in idempotent semimodules. Linear Algebra Appl. 379, 395-422 (2004)

11. Develin, M., Sturmfels, B.: Tropical convexity. Doc. Math. 9, 1-27 (2004)

12. Gondran, M., Minoux, M.: L'indépendance linéaire dans les dioïdes. Bull. Dir. Etudes Rech. EDF, Sér. C (1978)

13. Gondran, M., Minoux, M.: Linear algebra in dioids: a survey of recent results. In: Algebraic and Combinatorial Methods in Operations Research. North-Holland Math. Stud., vol. 95, pp. 147-163. North-Holland, Amsterdam (1984)

14. Gaubert, S., Sergeev, S.: Cyclic projectors and separation theorems in idempotent convex geometry. Fund. Prikl. Mat. 13, 33-52 (2007). In Russian, Engl. translation in Journal of Mathematical Sciences (Springer, New York), Vol. 155, No. 6, pp. 815-829, 2008

15. Helbig, S.: On Caratheodory's and Kreĭn-Milman's theorems in fully ordered groups. Comment. Math. Univ. Carol. 29(1), 157-167 (1988)

16. Joswig, M., Sturmfels, B., Yu, J.: Affine buildings and tropical convexity. Alban. J. Math. 1(4), 187211 (2007)

17. Lindström, B.: A theorem on families of sets. J. Comb. Theory 13, 274-277 (1970)

18. Litvinov, G.L., Maslov, V.P., Shpiz, G.B.: Idempotent functional analysis: an algebraic approach. Math. Notes 69(5), 696-729 (2001)

19. Maslov, V.P.: Méthodes Operatorielles. Mir, Moscow (1987)

20. Matoušek, J.: Lectures on Discrete Geometry. Springer, Berlin (2002)

21. Mikhalkin, G.: Enumerative tropical algebraic geometry in $\mathbb{R}^{2}$. J. Am. Math. Soc. 18(2), 313-377 (2005)

22. Nitica, V., Singer, I.: Max-plus convex sets and max-plus semispaces. I. Optimization 56(1-2), 171205 (2007)

23. Plus, M.: Linear systems in (max, + )-algebra. In: Proceedings of the 29th Conference on Decision and Control, Honolulu, December 1990

24. Richter-Gebert, J., Sturmfels, B., Theobald, T.: First steps in tropical geometry. In: Idempotent Mathematics and Mathematical Physics. Contemp. Math., vol. 377, pp. 289-317. Am. Math. Soc., Providence (2005)

25. Reutenauer, C., Straubing, H.: Inversion of matrices over a commutative semiring. J. Algebra 88(2), 350-360 (1984)

26. Sarkaria, K.S.: Tverberg's theorem via number fields. Isr. J. Math. 79, 317-320 (1992)

27. Sturmfels, B., Zelevinsky, A.: Maximal minors and their leading terms. Adv. Math. 98(1), 65-112 (1993)

28. Tverberg, H.: A generalization of Radon's theorem. J. Lond. Math. Soc. 41, 123-128 (1966)

29. Tverberg, H.: On equal unions of sets. In: Studies in Pure Mathematics, pp. 249-250. Academic Press, London (1971)

30. Viro, O.: Dequantization of real algebraic geometry on logarithmic paper. In: European Congress of Mathematics, vol. I, Barcelona, 2000. Prog. Math., vol. 201, pp. 135-146. Birkhäuser, Basel (2001)

31. Zimmermann, K.: A general separation theorem in extremal algebras. Ekon.-Mat. Obz. 13(2), 179201 (1977) 